\title{
Self-imaging technique for beam collimation
}

\author{
Luis Miguel Sanchez-Brea, ${ }^{1, *}$ Francisco Jose Torcal-Milla, ${ }^{1}$ Jose Maria Herrera-Fernandez, ${ }^{1}$ \\ Tomas Morlanes, ${ }^{2}$ and Eusebio Bernabeu ${ }^{1}$ \\ ${ }^{1}$ Optics Department, Applied Optics Complutense Group, Universidad Complutense de Madrid, \\ Facultad de Ciencias Físicas, Ciudad Universitaria s.n., 28040, Madrid, Spain \\ ${ }^{2}$ AOTEK, Barrio de San Andrés, s.n., 20500, Mondragón, Guipúzcoa, Spain \\ *Corresponding author: sanchezbrea@fis.ucm.es
}

Received July 30, 2014; accepted August 31, 2014;

posted September 4, 2014 (Doc. ID 220041); published 0 MONTH 0000

\begin{abstract}
A simple collimation technique based on measuring the period of one self-image produced by a diffraction grating is proposed. Transversal displacement of the grating is not required, and then automatic single-frame processing can be performed. The self-image is acquired with a CMOS camera, and the period is computed using the variogram function. Analytical and experimental results are obtained, which show the simplicity and accuracy of the proposed technique. ( 2014 Optical Society of America

OCIS codes: (050.1940) Diffraction; (050.1950) Diffraction gratings; (070.6760) Talbot and self-imaging effects; (120.1680) Collimation.
\end{abstract}

http://dx.doi.org/10.1364/OL.99.099999

Optical beams with high collimation degree are required in numerous optical applications such as metrology and information processing systems. The simplest technique is auto-collimation, where the size of the beam is compared at two different distances from the source. Nevertheless, more accurate collimation techniques based on self-imaging and interferometry have been developed to obtain a greater collimation degree [1-8]. In addition, dynamic detection method using moiré fringes and grating transverse displacement is proposed in $[\underline{9}, 10]$. On the other hand, a technique based on double-grating imaging is shown in [11]. In this technique, the optical beam with wavelength $\lambda$ passes through a diffraction grating with period $p$. A mask composed by two gratings with the same period $p$ and displaced laterally a distance $p / 4$ between them is located at a Talbot plane. Two photodetectors are placed just behind each grating. When the beam is collimated, both signals are shifted $90^{\circ}$, and the Lissajous figure results a circle (considering equal amplitude). When the beam is not collimated, the phase shift between the signals is not exactly $90^{\circ}$, and the Lissajous figure becomes an ellipse. The collimation degree can be determined automatically by measuring the phase shift between both signals.

This technique has been proven robust and accurate. However, a continuous transversal displacement of the grating is required in order to obtain the Lissajous figure. Another technique for beam collimation has been proposed recently, where transversal displacement is not necessary [12]. In this case parabolic fringes are obtained that inform us about the collimation degree by using two gratings, one circular and another linear. Nevertheless the numerical analysis of the fringes to determine the collimation degree is neither simple nor clear.

In this work, we propose a simple and accurate collimation technique based on the self-imaging phenomenon which only requires a diffraction grating and a linear array of photodetectors. Transversal displacement of the grating is not required, and then automatic single-frame processing can be performed. The collimation of the beam is achieved by comparing the period of one selfimage with that of the grating until both periods are equal. We can collimate accurately small-size light beams with this method, provided that Talbot self-images are obtained. In addition, this collimation technique can also be performed for polychromatic light sources, since an achromatic self-imaging regime is also possible [13].

Let us consider the setup shown in Fig. 1. It consists of a point source of wavelength $\lambda$, a lens of focal length $f$, a diffraction grating $G$, and a CMOS camera. The diffraction grating with period $p$ is placed at a distance $z_{1}$ from the lens. The transmittance of the grating is $t(x)=$ $\sum_{n} a_{n} \exp (i q n x)$ being $n$ integer numbers, $a_{n}$ are the Fourier coefficients of the grating, and $q=2 \pi / p$. For amplitude gratings, fringes of the same period as the grating period with maximum contrast are observed at distances $z_{2}=l z_{T}$, where $z_{T}=2 p^{2} / \lambda$ is the Talbot distance, and $l$ is an integer. When the emitter is exactly placed at the focal point, $\Delta z=\left|z_{0}-f\right|=0$, the beam after the lens is properly collimated, and the period of the self-images is equal to that of the grating. When the emitter is not exactly at the focal point, $\Delta z \neq 0$, the intensity distribution at $z_{2}$ results in [11]

$$
I\left(x_{3}, z_{2}\right) \propto I_{0} \sum_{n, n^{\prime}} a_{n} a_{n^{\prime}} e^{i \frac{q}{1+\alpha z_{2}}\left(n-n^{\prime}\right) x_{3}} e^{-i \frac{q^{2}}{2 k}\left(n^{2}-n^{\prime 2}\right) \frac{z_{2}}{1+\alpha z_{2}}},
$$

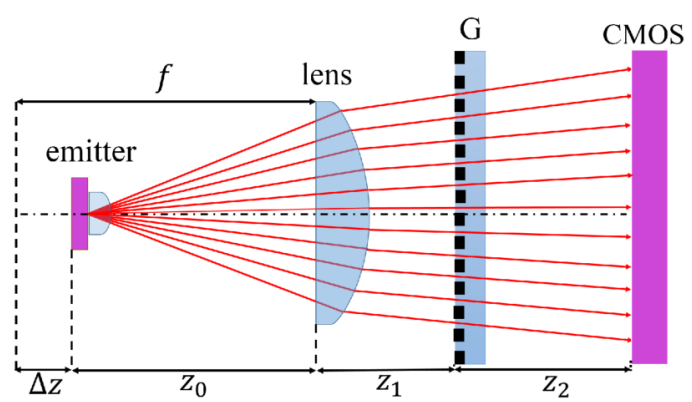

Fig. 1. Experimental setup for beam collimation. $z_{0}$ is the distance from emitter to the lens, whose focal length is $f, z_{1}$ is the distance from the lens to the diffraction grating $G, \Delta z=z_{0}-f$ is the distance from the emitter to the focal point of the lens, and $z_{2}$ is the distance from the grating to the observation plane, where a CMOS camera is placed. 
80 where $x_{3}$ is the coordinate parallel to the grating and
81 perpendicular to the fringes, $I_{0}$ is the intensity of the incoming beam, $k=2 \pi / \lambda$, and $\alpha \approx-\Delta z / f^{2}$. The period of the fringes $p_{\Delta z}$ is obtained from the first exponential term,

$$
p_{\Delta z}=\left(1+\alpha z_{2}\right) p .
$$

where it depends on the distance $\Delta z$ from the source to the focal point. On the other hand, the second exponential term indicates the location of the self images,

$$
z_{T, \Delta z}=\left(1+\alpha z_{2}\right) z_{T}
$$

which are slightly separated when the beam is not collimated.

To measure the collimation of the beam, the best option is (2) since it is only required the intensity distribution at Talbot distance. Reorganizing Eq. (2) $\Delta z$ results in

$$
\Delta z=-\frac{f^{2}}{z_{2} p} \Delta p,
$$

where $\Delta p=p_{\Delta z}-p$ is the variation of the self-image period with respect to that of the grating. For a certain set-up, the focal distance of the lens $f$, the period of the grating $p$, and the distance from the grating to the observation point $z_{2}$ are fixed, and the only parameter that varies with the collimation of the beam is the period of the fringes at the observation plane $p_{\Delta z}$.

For the experimental set-up depicted in Fig. 1, we have used a diffraction grating with period $p=10 \overline{0} \mu \mathrm{m}$. The manufacturing error of the grating is $3 \mu \mathrm{m} / \mathrm{m}$, which corresponds to $0.3 \mathrm{~nm} /$ period. The laser diode is HE8807SG by Hitachi with wavelength $\lambda=880 \mathrm{~nm}$, and collimation is performed along the axis perpendicular to the fringes of the grating. We use a collimation lens with focal length $f=25 \mathrm{~mm}$ and diameter $D=20 \mathrm{~mm}$. Self-images are acquired with a bidimensional CMOS camera DMK72BUC02 by Imaging Source whose pixel size is $2.2 \mu \mathrm{m}$. We have only used one row of the camera, and for a final set-up, a linear array of photodetectors can be a simpler option. The CMOS camera is placed at a distance $z_{2}=z_{T}=22.7 \mathrm{~mm}$ from the grating. The grating and the CCD camera have been carefully aligned to the optical axis. All elements are fixed except the laser diode, which is displaced along the optical axis with a step by step motorized stage.

The self-images obtained for several positions of the laser diode are shown in Fig. 2. The step in the displacement is $100 \mathrm{~nm}$. We can see how the period of the fringes increases, and the intensity of the signal decreases when the laser diode position $\Delta z$ is varied. As an example, a zoom of the self-image profile for $\Delta z+c=7.5 \mathrm{~mm}$ is shown in Fig. 3(a). Due to experimental conditions the self-images are not perfectly uniform and present random irregularities. These irregularities complicate a proper period estimation. As a consequence, we have used the variogram function defined as [14]

$$
2 \gamma(h)=\left\langle[I(x+h)-I(x)]^{2}\right\rangle_{x},
$$

where $h$ is the distance between any two data of the signal, and $\langle\bullet\rangle_{x}$ means averaging with respect to $x$, to

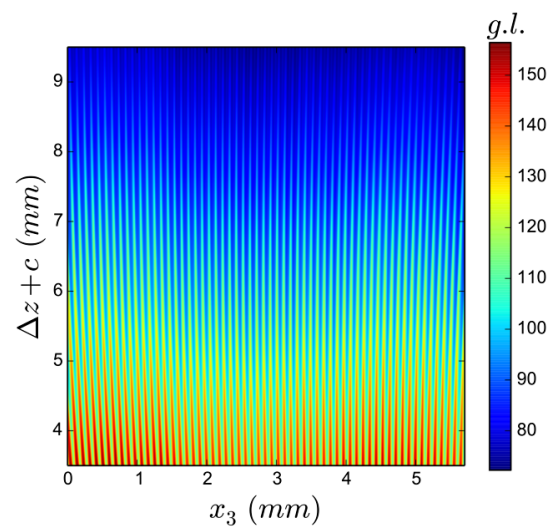

Fig. 2. Experimental fringes for different locations of the laser diode along to the $z$-axis (grating period $p=100 \mu \mathrm{m}$ and laser diode wavelength $\lambda=880 \mathrm{~nm}$ ). $c$ represents a constant value given by the initial position of the linear stage, which is unknown.

filter random fluctuations in the signal. The variogram function has been used previously for fringe quality improvement $[15,16]$. Since each datum of the variogram is obtained by an averaging, it is much smoother than the original signal. In addition, the variogram of a periodic signal is also periodic with the same period. As an example, the variogram for the profile of Fig. 3(a) is shown in Fig. 3(b). Therefore, we can determine the period of the signal by measuring the period of the variogram, with the advantage of eliminating random fluctuations and noise. When the signal is sinusoidal, $I(x)=A+B$ $\sin (q x+\delta)$, the variogram function results in $2 \gamma(h)=$ $2 B^{2} \sin ^{2}(q h / 2)=B^{2}[1-\cos (q h)]$, where $B$ is the amplitude of the signal. Due to signal irregularities, the experimental variogram is better fitted to

$$
2 \gamma(h)=(\alpha+\beta x)-(\gamma+\varepsilon x) \cos (2 \pi h / p),
$$

where $\alpha, \beta, \gamma, \varepsilon$, and $p$ are free parameters. The parameters $\alpha, \beta, \gamma$, and $\varepsilon$ can be easily measured from the experimental variogram. In a first attempt, we have used the scipy.optimize toolbox in Python (www.scipy.org) in order to obtain the period of the signal for each distance $\Delta z$. The method that has produced best results is the minimize_scalar function [17], where we obtained a standard deviation of $1.5 \mathrm{~nm}$ in the period estimation. Nevertheless, this technique is quite time consuming, approximately $6 \mathrm{~s}$ for each profile in a Intel Core i7 processor. As a consequence, for a real-time collimation technique, we have opted for measuring the location of the variogram minima by means of a quadratic fitting, since they are quite parabolic [Fig. $3(\mathrm{~b})$ ]. In the example, we have computed the position for each minimum up to the ninth, and then we have performed a linear fitting $y=p n$, where $n$ is the order of the minimum, Fig. 3(c). The period is obtained as the slope of the fitting. The processing time for the period estimation was $0.6 \mathrm{~s}, 10$ times faster.

The difference $\Delta p=p_{\Delta z}-p$ between the experimental period $p_{\Delta z}$, obtained for each distance $\Delta z$ with this linear fitting, and the period of the grating $p$ is shown in Fig. $\underline{4}$ (red line). It has been performed for 300 measurements along $30 \mu \mathrm{m}$ of displacement. The histogram of the
F2:1

F2:2

$\mathrm{F} 2: 3$

F2:4

F $2: 5$ 
(a)

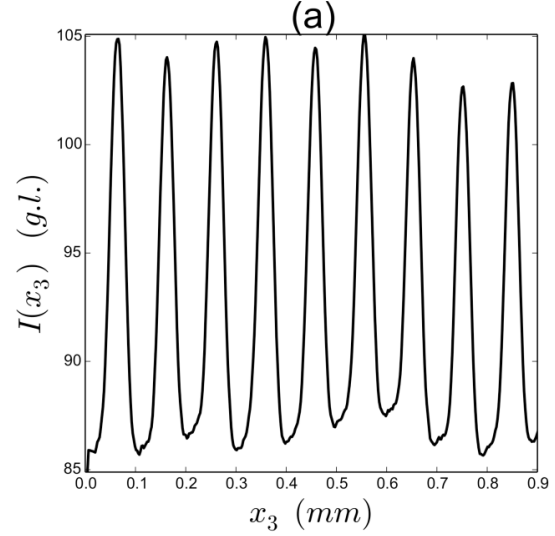

(b)

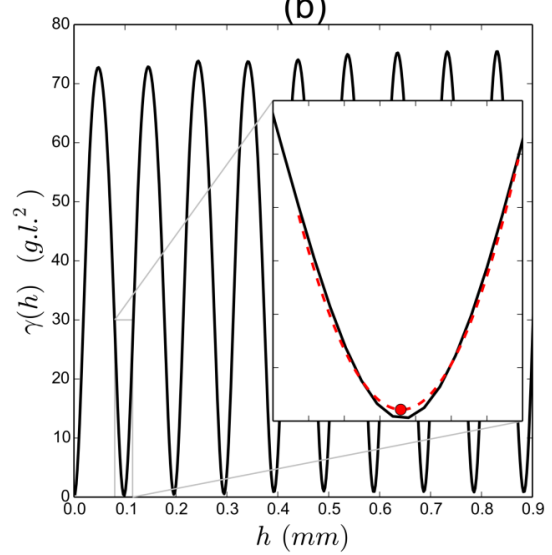

(c)

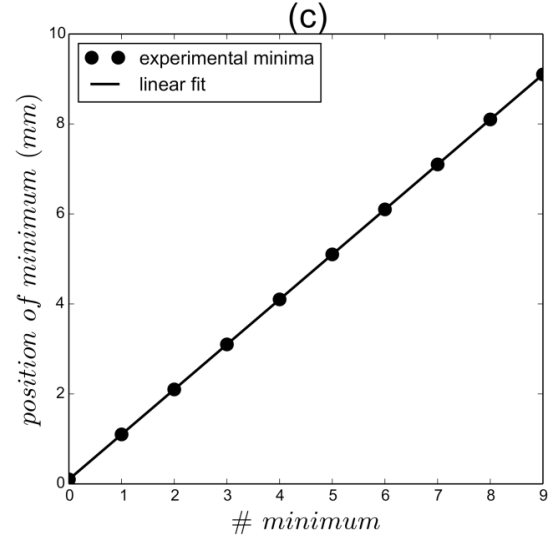

Fig. 3. (a) Section of the self-image profile of Fig. 2 for $\Delta z+c=7.5 \mathrm{~mm}$. (b) Semi-variogram computed with this signal. The variogram minima are fitted to a parabolic profile (dashed line in zoom). Each minimum in the parabolic fitting (red dot) is used in Fig. 3(c) for period estimation. (c) Linear fitting of the variogram minima. The computed period is the slope of the fitting.

residuals, $r=\Delta p-(a \Delta z)$, presents a standard deviation $\delta p=\operatorname{std}(r)=0.56 \mathrm{~nm}$, and it is shown in Fig. 5(a). This standard deviation is comparable to the fluctuations of period of the grating due to manufacturing errors (approximately $0.3 \mathrm{~nm} /$ period). With this simple algorithm, we obtain even a better result than when all the variogram profile is fitted to (6), with minimize_scalar function $(1.5 \mathrm{~nm})$.

Finally, we have determined the standard deviation of the residuals in terms of the number of variogram minima considered in the linear fitting, Fig. 3(c). In Fig. 5(c), the

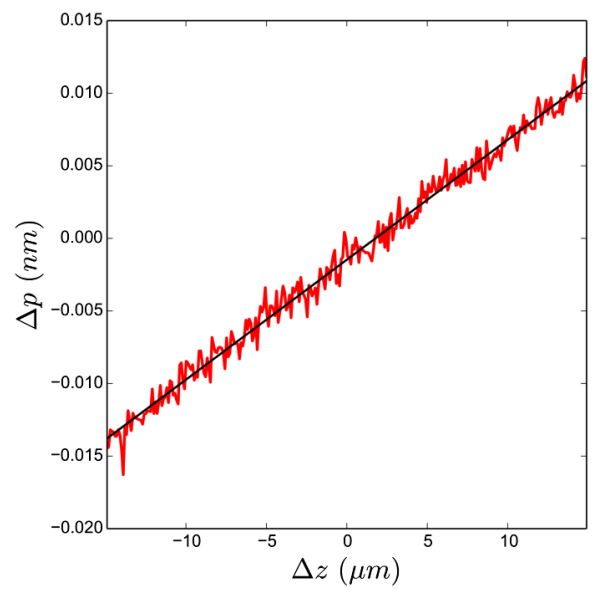

Fig. 4. Difference between the period of the self-image with respect to that of the grating, $\Delta p$, in terms of $\Delta z$ when the first 10 variogram minima have been used for period estimation (red line). Linear fitting to these data $\Delta p=a \Delta z$, where $a=0.00083$ (black line).

standard deviation of the residuals is shown, which decreases approximately as $1 / N$ by increasing the number of variogram minima considered in the linear fitting. On the other hand, when the number of variogram minima is reduced the algorithm is faster. For example, for $N=4$ the computation time is $0.16 \mathrm{~s}$, and for $N=2$ it is only $0.05 \mathrm{~s}$. For this last case, $\delta p$ is similar to that obtained with minimize_scalar function $(1.5 \mathrm{~nm})$ and 120 times faster.

(a)

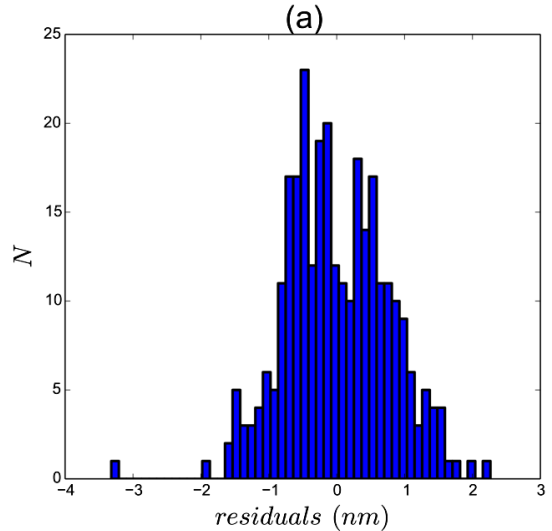

(b)

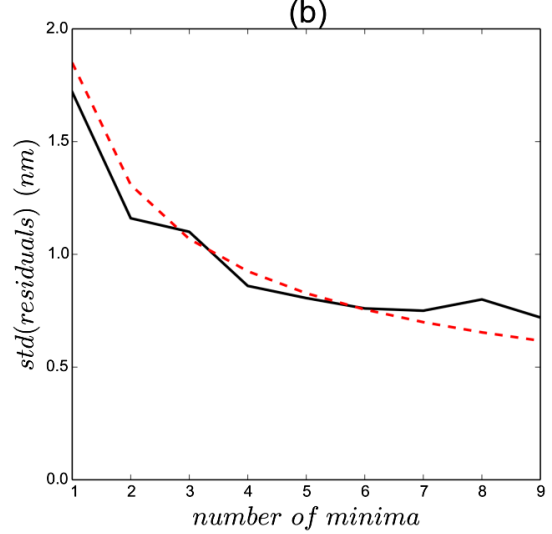

Fig. 5. (a) Histogram of residuals for data of Fig. 4 when 10 variogram minima are used for the period estimation. (b) Standard deviation of residuals in terms of the number of variogram minima considered for linear fitting.
F4:1

F4:2

F4:3

F $4: 4$

F $4: 5$

183

184

185

186

187

188

189

190

191 
Finally, since the relationship between position and period is linear, we can easily determine the resolution in the divergence of the collimated beam. The resolution in the location of the emitter results $\delta z=\delta p / a=$ $0.67 \mu \mathrm{m}$, where $a$ is the slope of Fig. 4 . These results are similar to other recent techniques [18-20], with the advantage of a simpler experimental realization. Considering the lens equation, $1 / s^{\prime}-1 / s=1 / f^{\prime}$, with $s=-f+\delta z$, we obtain that the minimum observable beam divergence is $\delta \phi=D / 2 s^{\prime} \approx D \delta z / 2 f^{\prime 2}$. Since we have used a doublet lens with diameter $D=20 \mathrm{~mm}$ and focal length $f=25 \mathrm{~mm}$, the resolution in the divergence of the light beam results $\delta \phi=10 \mu \mathrm{rad} \approx 0.0006^{\circ}$.

In conclusion, we propose a simple technique for beam collimation that only uses one diffraction grating and one linear array of photodetectors. No lateral displacement of the grating is required. In addition processing of the signal is very simple since we only have to compute the period of the self-image captured with the photodetector. To reduce the irregularities of the self-image fringes, we have used the variogram function. Instead of using a optimization algorithm for the period computation, we have performed a parabolic fitting of the variogram minima. As a consequence, we have reduced the computation time up to two orders of magnitude. With the proposed algorithm we have obtained an experimental divergence of $10 \mu \mathrm{rad}$ for a doublet lens with diameter $D=20 \mathrm{~mm}$ and focal length $f=25 \mathrm{~mm}$.

The authors thank to Jose Luis Vilas for his valuable comments. This work has been supported by the Ministry of Science and Innovation of Spain (project DPI201127851) and project Art. 83 LOU "Estudio de tecnología para la realización de codificadores ópticos de $2 \mu \mathrm{m}$ de periodo" with Fagor Automation S. Coop.

\section{References}

1. M. V. R. K. Murthy, Appl. Opt. 3, 531 (1964).

2. D. Malacara, Optical Shop Testing, Chap. 4 (Wiley, 1978).

3. A. R. Ganesan and P. Venkateswarlu, Appl. Opt. 32, 2918 (1993).

4. D. E. Silva, Appl. Opt. 10, 1980 (1971).

5. D. Joyeux and Y. Cohen-Sabban, Appl. Opt. 21, 625 (1982).

6. J. Choi, G. Perera, M. Aggarwal, R. Shukla, and M. Mantravadi, Appl. Opt. 34, 3628 (1995).

7. J. S. Darlin, M. P. Kothiyal, and R. S. Sirohi, J. Mod. Opt. 45, 2371 (1998).

8. P. Senthilkumaran, Appl. Opt. 42, 6314 (2003).

9. S. Yokozeki, K. Patorski, and K. Ohnishi, Opt. Commun. 14, 401 (1975).

10. S. Haramaki, S. Yokozeki, A. Hayashi, and H. Suzuki, Proc. SPIE 4416, 388 (2001).

11. L. M. Sanchez-Brea, F. J. Torcal-Milla, F. J. SalgadoRemacha, T. Morlanes, I. Jimenez-Castillo, and E. Bernabeu, Appl. Opt. 49, 3363 (2010).

12. K. Patorski, K. Pokorski, and M. Trusiak, Opt. Lett. 39, 291 (2014).

13. N. Guerineau, B. Harchaoui, and J. Primot, Opt. Commun. 180, 199 (2000).

14. N. Cressie, Statistics for Spatial Data, revised ed., vol. 928 (Wiley, 1993).

15. L. M. Sanchez-Brea, F. J. Torcal-Milla, and E. Bernabeu, Appl. Opt. 46, 5027 (2007).

16. L. M. Sanchez-Brea and E. Bernabeu, Appl. Opt. 44, 3276 (2005).

17. http://docs.scipy.org/doc/scipy/reference/generated/scipy optimize.minimize_scalar.html.

18. J. Dhanotia and S. Prakash, Appl. Opt. 50, 1446 (2011).

19. R. Disawal, J. Dhanotia, and S. Prakash, Prec. Eng. 1 (in press).

20. W. Y. Chang, K. Y. Hsu, K. H. Chen, and J. H. Chen, Opt. Lasers Eng. 62, 126 (2014). 


\section{Queries}

1. AU: Please provide a status update for Ref. [19] if available 